\title{
Ser periodista en Asturias entre dos guerras, $1898-1936$
}

\author{
Víctor RODRÍGUEZ INFIESTA \\ rodriguezvictor@uniovi.es \\ Universidad de Oviedo
}

Recibido: 21 de julio de 2014

Aceptado: 19 de enero de 2015

\section{Resumen}

El artículo analiza la configuración del oficio de periodista en las primeras décadas del siglo XX a través del caso asturiano. Para ello se utiliza una selección de la bibliografía y las fuentes disponibles, relacionando esta información con otros espacios. La evolución desde principios de siglo es evidente, aunque limitada, como puede comprobarse a través de algunos datos sobre salarios, la evolución de las plantillas o, entre otras cosas, la creación de las primeras asociaciones de la prensa.

Palabras clave: Profesionales del periodismo, Asturias, Primer tercio del siglo XX, prensa.

\section{Being a Journalist in Asturias in between Two Wars, 1898-1936}

\begin{abstract}
The article analyzes the configuration of the profession of journalism in the early decades of the twentieth century, using the Asturian case by means of example. To this analysis, a selection of the available literature and sources are used to relate this information with that of other territories. Developments since the turn of the century are clear, though limited, as can be seen through some wage data, the evolution of the number of staff or, amongst other things, the creation of the first Press associations.
\end{abstract}

Keywords: Professional journalists, Asturias, the first third of the twentieth century, press.

\section{Referencia normalizada}

RODRÍGUEZ INFIESTA, Víctor (2015): "Ser periodista en Asturias entre dos guerras, 1898-1936". Estudios sobre el Mensaje Periodístico. Vol. 21, Núm. 1 (enero-junio), págs.: 177-192. Madrid, Servicio de Publicaciones de la Universidad Complutense.

Sumario: 1. Introducción. 2. Salarios y condiciones de trabajo. 3. ¿Una verdadera profesión?. 4. Ser periodista, ¿para qué? 5. Conclusiones. 6. Referencias bibliográficas.

\section{Introducción}

Los procesos de configuración del oficio de periodista siguen siendo un terreno poco cultivado en la historia de la comunicación social en España, pese a la existencia de algunos trabajos imprescindibles. Los estudios regionales deberían ayudarnos a construir una imagen más completa de los distintos ritmos de evolución que se dan en la península, resultando particularmente interesante el estudio de espacios como el asturiano, hasta hace pocos años escasamente atendido por los investigadores pese a su relevancia. Los años que transcurren entre 1898 y 1936 configuran una época de lento despegue hasta la Guerra Civil y sus consecuencias, cuando se derrumban muchos de los elementos de modernización sobre los que estaba construyéndose la sociedad española. Se trata, en Asturias, de un periodo particularmente rico en la historia del periodismo, con una evolución acelerada de distintos factores y la presencia de cabeceras tan influyentes como El Comercio (1878-), El Noroeste (1897-1936), El Correo de Asturias (1890-1922), El Carbayón (1879-1936), La Voz de Asturias (1923-2012), La Prensa (1921-1936), La Voz de Avilés (1908-), Región (1923-1983) y otras. Podría 
hablarse de una edad de oro del periodismo asturiano, por la calidad de muchos de los periódicos que se editan en la región, por su influencia en Asturias y también por su proyección en el exterior. Para su análisis se ha combinado la consulta, en el capítulo de las fuentes primarias, de bibliografía y testimonios de época, prensa e información de archivo, siempre con el objeto de aproximarnos a un tema de estudio que, pese a condicionar la producción de contenidos y mensajes periodísticos, permanece casi inédito en Asturias ${ }^{1}$.

Son años en los que los que los contornos y las condiciones laborales del oficio van definiéndose lentamente. Y es que, como en el resto de España, en la Asturias de principios del siglo XX los periódicos seguían haciéndose solos, sin necesidad de periodistas. Éstos, sí, acudían a las redacciones algunas noches y allí velaban las armas de su singular oficio, envueltos en una atmósfera que describe Julio Camba:

"Ordinariamente, los redactores nos reuníamos en torno a una mesa muy grande, pedíamos café y comenzábamos a charlar y a fumar pitillos. Abajo estaban los talleres. ¿Por qué procedimiento se transformaban nuestras conversaciones en artículos y noticias? Yo lo ignoro; pero el hecho es que el periódico, poco a poco, iba haciéndose" (Camba, "Los periódicos se hacen solos", artículo publicado en El Sol, 5 de diciembre de 1919, sección "Crónicas de Camba", p.1 [recogido en Camba, 1946])

Otras veces ni siquiera aquello, porque los redactores, probablemente atrapados en otros atractivos de la vida nocturna, no se molestaban en hacer acto de presencia. Y pese a todo "la rotativa giraba, y el periódico salía, y hasta es posible que saliera mejor que nunca..." (Camba, íbidem).

El periodista gallego lo dejó esbozado en el citado artículo con su humor característico, aunque los testimonios directos de la falta de profesionalidad con la que durante mucho tiempo se elaboraron algunos periódicos -tijeretazos, refritos, fabulaciones y otras habilidades- darían para llenar un grueso volumen. Un periodista mierense, Antonio Álvarez Solís, además de referirse varias veces a los tiempos en los que los periódicos "se hacían solos a fuerza de tomar café" (atribuyéndole la expresión a Pérez Lugín) y de señalar que en Asturias aquello ya iba quedando atrás cuando se inició en el periodismo, esto es, hacia los años veinte, relata una anécdota que ilustra la base real de las ocurrencias de Julio Camba. Se trataba de cómo, ante la ausencia de todos los redactores de El Carbayón por ser incapaces de llegar a tiempo a Oviedo tras una excursión, el regente de la imprenta había sacado a la calle el número del día siguiente con la mayor facilidad, valiéndose únicamente de unas tijeras y algunos periódicos de otros lugares recibidos al cambio (Álvarez Solís, 1946: 18 y ss.)².

Los años que aquí nos ocupan son, no obstante, una etapa de transición hacia un ejercicio profesional cada vez más regulado, disolviéndose a la larga los aspectos más chocantes de aquellos periódicos del pasado. No es lo mismo ser periodista en la Asturias de principios de siglo que en la Asturias de la República, entre otras cosas porque entremedias se ha consolidado el periodismo de empresa y la proverbial desunión

1 Como una notable excepción puede citarse el trabajo de Gabriel Santullano, 2004.

2 Una referencia similar puede verse en Constantino Cabal (1941:35), quien señala cómo a veces El Carbayón lo hacía Ramón “el de la máquina”. 
reinante ha dejado paso a un asociacionismo que, mal que bien, logra paliar determinadas situaciones. Por supuesto, no hay en el hecho de "ser periodista" una categoría única, comenzando a establecerse el corte principal entre profesionales y no profesionales, algo que, como se verá más adelante, tiene también su reflejo en el despertar al asociacionismo de los periodistas asturianos. Y por otra parte, no todo cambia en estos años. Los mecanismos de control y la exposición a la violencia de distinto tipo han sido unas de las características permanentes del ejercicio del periodismo hasta el presente. Que los periódicos se hicieran "solos" no significa que la máquina de la represión girara en el vacío; por el contrario descargaba sus golpes, y con particular virulencia en algunos de los momentos analizados. Conviene recordarlo, aunque los límites de este artículo no permitan más que apuntar cuestiones de este tipo.

\section{Salarios y condiciones de trabajo}

Al margen de las actividades de aquellos escritores que contemplan su labor periodística más como un compromiso con la causa defendida que como un oficio, parece razonable adentrarse en el mundo laboral de la prensa asturiana mediante una aproximación a un elemento inseparable de toda actividad realmente profesional: el cobro de los servicios prestados. A principios del siglo XX el panorama sigue pareciéndose todavía mucho al de la centuria pasada, con salarios que no permiten vivir dignamente, periódicos que siguen haciéndose prácticamente por una sola persona, meritorios, sablistas impenitentes, trabajadores que no consiguen cobrar.... O anécdotas como la protagonizada por el administrador de un periódico ovetense que, a la vista de una caja totalmente vacía y enternecido por las súplicas de determinado redactor que "mendigaba" el fruto de su trabajo, concluyó por enviarle al lugar donde estaba el mejor vendedor del periódico para que le diera unas monedas (Álvarez Solís, 1946: 18-19).

Después la situación mejora, pero nunca demasiado. Periódicos hay en 1920 cuya suspensión temporal se justifica por un viaje de su director ${ }^{3}$; y periodistas en torno a las mismas fechas que encabezan cartas dirigidas a los responsables del periódico con expresiones alarmantes: "nuevamente tengo que recurrir a su amistad", para seguir avanzando: "creí que aún había dinero en casa, pero me entera mi esposa que el remanente se fue..." y llegar al punto neurálgico: "así que si puede facilitarme un préstamos de 200 pts se lo agradeceré vivamente"4.

Los salarios que se cobran en Asturias son reflejo del resto de la prensa española, teniendo en cuenta las diferencias internas según los puestos ocupados y que Madrid es en todo momento un caso singular. Entre 100 y 125 pesetas mensuales se situarían los sueldos en la capital española a principios de siglo como media aproximada, mientras los periódicos de empresa hicieron que abundaran los sueldos en torno a las 150 pesetas desde entonces hasta 1920 (Desvois, 1996; Seane y Sáiz, 1996: 44-48). Referirse a sueldos medios, de todos modos, implica recortar una realidad cuyos verdaderos perfi-

3 Por ello y por falta de papel anunciaba El Progreso de Asturias, de Avilés, la suspensión momentánea de la edición (El Carbayón, 6-10-1920).

4 Fragmentos de una carta personal con membrete de El Comercio, fechada el 11 de noviembre de 1918 y dirigida por M. Vega a Joaquín Alonso Bonet, Museo del Pueblo de Asturias (en adelante $M P A$ ), Gijón, fondos procedentes del archivo de J. Alonso Bonet. 
les dibujan caprichosas formas. José López Pinillos (Alma Española, 31-1-1904) aludía al pago de 30 duros (150 pesetas) para el articulista encargado de los fondos editoriales en un periódico modesto, descendiendo a partir de aquí la escala de los jornales: "Un literato que escribía con intención y gracia, lo mismo de literatura y de música que de política y de toros, cobraba veinticinco. Otro que hilvanaba críticas, componía crónicas, inflaba telegramas y enjaretaba revistas parlamentarias, veinte, y los demás quince, doce y diez". Por la parte de arriba, según el mismo escritor, los grandes periódicos no pagaban sueldos de hambre, de cincuenta pesetas, pero sí abundaban los de cien y ciento cincuenta pesetas, escaseaban los de quinientas, "y antes que a embolsarse mil, puede llegar un redactor a encasquetarse la tiara y gobernar todo el orbe católico". Limitaciones evidentes, por lo tanto, pero también la posibilidad de sobresalir en algún momento. En 1922 los sueldos de 300 pesetas abundaban entre los redactores de $\mathrm{El} \mathrm{Sol}$, diario que representa un importante impulso modernizador en cuanto al ejercicio del periodismo en España; y también se registran sueldos por encima de la cantidad indicada, hasta alcanzar las 750 pesetas mensuales del asturiano Fernando Vela ${ }^{5}$. Desde algún tiempo antes $A B C$, que también jugó un papel destacado en este sentido y que exigía como $\mathrm{El} \mathrm{Sol} \mathrm{de-}$ dicación exclusiva a sus redactores, venía pagando salarios dignos: 250 pesetas de partida en los primeros años (García Venero, 1961: 135).

Las cosas en Asturias eran un poco distintas. El primer sueldo de Joaquín Alonso Bonet al ingresar en el diario republicano El Publicador en 1909 fue de 75 pesetas, pasando a cobrar como redactor de calle antes de concluir el mismo año en $E l \mathrm{Co}$ mercio 125 pesetas mensuales, tal y como él mismo recordaba en una entrevista muchos años después (Voluntad, 19-1-1975) ${ }^{6}$. En el citado diario el sueldo de otro de los periodistas gijoneses más recordados, Alfredo García García (Adeflor), ascendía en torno a las mismas fechas -son nuevamente declaraciones del interesado en una entrevista- a 200 pesetas más una comisión ligada al crecimiento de la tirada (Gaceta de la Prensa Española, 7-1945: 1.603-1.608 ); pero lejos de ser como Bonet un joven recién desembarcado en el mundo del periodismo, Adeflor era ya por entonces un periodista realmente popular en Gijón, con algunos años de dedicación profesional a sus espaldas y que se situaba al frente de la redacción de El Comercio para relanzar en el mercado local al decano de la prensa diaria asturiana. Más elevado era el sueldo del director de La Opinión de Asturias en 1907: 320 pesetas (Santullano, 2004: 482). En todo caso, Jesús Evaristo Casariego (1972: 163) apunta todavía para 1910 un sueldo como promedio para los redactores de Oviedo de 75 pesetas. Y en general, lo común en la segunda década del siglo debió sobrepasar escasamente las 100 o 125 pesetas mensuales, cantidades que cobraban respectivamente dos de los redactores de El Noroeste en 1917, mientras el redactor jefe (lo era por entonces José Valdés Prida), recibía por su trabajo sólo un poco más: 150 pesetas. La llegada a la dirección del diario reformista en julio del mismo año de Antonio L. Oliveros parece ser que mejoró aquella situación, repartiendo 100 pesetas de su propio sueldo entre sus subalternos, según afirmaba él mismo (López Oliveros, 1935: 111).

5 "Personal de la redacción de El Sol". Estudios de Historia Social, n” 24-25, pp. 451-452 [documento procedente del archivo de Nicolás $\mathrm{M}^{\mathrm{a}}$ de Urgoiti].

6 Cfr. Casariego, 1990: 20-22. 
A primera vista los sueldos crecieron en los años veinte. En la nómina de redactores de un nuevo diario gijonés, La Prensa, los totales de los honorarios percibidos anualmente por distintos redactores arrojaban un desigual resultado, de modo que mientras en 1923 y 1924 algunos periodistas cobraron una media mensual ligeramente por encima de las 100 pesetas otros casi triplicaron tal cantidad e incluso superaron las 300 pesetas $^{7}$. En esta última cifra y refiriéndose también a aquellos años situaba Francisco Arias de Velasco la cantidad que solían cobrar los primeros redactores de otro diario aún de corta vida, $L a$ Voz de Asturias, si bien él mismo aseguraba percibir 350 pesetas, probablemente "el sueldo más alto exceptuando el de redactor-jefe y director" (La Voz de Asturias, 23-10-1977). Sin embargo, e incluso aceptando que estas últimas cantidades constituyeran en los años veinte la nota común en Asturias, la mejoría debe reconsiderarse teniendo en cuenta la imparable inflación vinculada a la Guerra Europea, factor que contrarresta unos avances que sólo pueden apreciarse en su justo y reducido valor si se comparan los sueldos de los periodistas con los de otras profesiones ${ }^{8}$. Ser redactor de un periódico asturiano, en definitiva, siguió siendo incompatible en muchos casos con el cobro de un sueldo digno, situación que no difería excesivamente de la de otras provincias. J. M. Desvois (1996: 35) calcula que el sueldo medio de un redactor de plantilla en provincias varía a principios de siglo entre las 75 y las 100 pesetas. Esta última es la cantidad que ganaban en 1910 dos redactores de un periódico gallego, La Región (Valladares Fernández, 1996: 492); y el sueldo que comenzó a cobrar José Laguillo Bonilla (1979: 216) en el otro extremo de la península en torno a 1903, al ingresar en la redacción de El Liberal de Sevilla. Siguiendo con algunos datos únicamente a modo de ejemplo, los sueldos que conocemos de los redactores de distintos periódicos editados en Valencia, cuna de una importante actividad informativa, oscilaban en los años veinte entre las 125 y las 200 pesetas (Ríus Sanchís, 2000: 88). Para Cataluña, el gran foco de producción periodística después de Madrid, J. L. Gómez Mompart (1992: 65) calcula que los sueldos se situaban entre las 50 y las 100 pesetas en 1900 , entre 150 y 300 pesetas en 1915 y entre 190 y 375 pesetas en 1925.

En todo caso durante la Segunda República, junto a la consolidación de otros factores modernizadores, los sueldos siguieron subiendo. Éstos oscilaban al parecer, a la altura de 1936, entre 250 pesetas y 400 pesetas mensuales en los periódicos de Oviedo

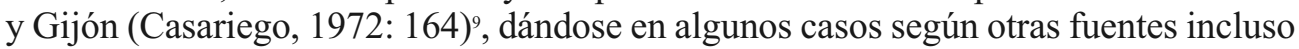

7 "Honorarios percibidos por los empleados del periódico La Prensa durante los años: 1924 1925 ", $M P A$, fondos J. A. Bonet. En el documento de indican las cantidades anuales cobradas por distintos redactores, resultando de la división entre los meses trabajados cantidades desiguales en las que tal vez pudiera incluirse el pago de alguna gratificación además del sueldo mensual. Estas son algunas de las cifras tal y como aparecen en el documento: Armando F. Buelta, 1924: 1.450 ptas. (sólo 11 meses); Faustino Sierra, 1923: 1.250 ptas.; 1924: 2.025 ptas.; Juvenal Iglesias, 1923: 2.100 ptas. (sólo 9 meses); Bernardo L. Torrens, 1923: 3.175 ptas.; 1924: 3.375 ptas.; Agustín Arias, 1923: 3.750 ptas.; 1924: 4.025 ptas.

8 Pueden verse algunos datos respecto al movimiento de salarios y precios en la Asturias del primer tercio del siglo XX en: Radcliff, 2004: 86-87 y 329-336.

9 En 1932 el propio autor comenzó a ganar en el diario Región 300 pesetas. 
salarios superiores. La pervivencia de prácticas tan arraigadas como era la dedicación a distintas actividades laborales, unida a la percepción de sueldos relativamente elevados, abrieron la puerta en algunos casos a situaciones como la que describe con júbilo Santiago Blanco (1977: 164-165) al recordar que en 1936 cobraba 500 pesetas como redactor del rotativo socialista ovetense Avance, 100 pesetas más como colaborador de otro diario de la misma orientación, La Tarde, más otros ingresos ("un enchufe de secretario de un jurado mixto" y algún dinero como defensor de trabajadores en el Tribunal Industrial), lo que sumaba "un sueldo fabuloso" cercano a las 1.000 pesetas.

Había, indudablemente, grandes diferencias en los sueldos, distintas categorías y por lo general un trato individualizado entre los redactores de un mismo periódico, pero además estaban las considerables diferencias salariales que afectaban a aquellos que no eran redactores. Dejando de lado a los colaboradores, que cuando cobraban solían hacerlo por artículo ${ }^{10}$, el escalón más bajo lo ocuparían los corresponsales en pequeñas poblaciones. A menudo éstos eran simples espontáneos o bien corresponsales administrativos que por la proximidad al periódico actuaban también como corresponsales literarios. En otras ocasiones se trataba de una actividad ligada a un salario, pero tan mísero que rara vez podía tomarse más que como una compensación por una actividad realizada con cierto desprendimiento y en paralelo a otras ocupaciones más lucrativas. A 30 pesetas mensuales ascendía la cantidad que un diario gijonés le señaló a Antonio Álvarez Solís, hacia los inicios de los años veinte, como corresponsal en un Mieres por entonces en plena ebullición. No llegó nunca a cobrarlas, y por otra parte le parecía entonces "que el dinero prostituía el noble oficio de periodista". Y no obstante, no tardó en pasar a ocupar el mismo puesto en El Carbayón, cobrando ahora 40 pesetas todos los meses, mientras desempeñaba al mismo tiempo otro trabajo en una empresa minerometalúrgica (Álvarez Solís, 1946: 14-16).

Sólo los corresponsales desplazados ocasionalmente fuera de Asturias percibieron sueldos más enjundiosos, así como los periodistas asturianos que complementaban sus ingresos con la corresponsalía de algún diario madrileño. Junto a los sueldos más o menos modestos se cobraron gratificaciones relacionadas con el número de telegramas cursados; era lo habitual en Oviedo, y sin duda también en otras ciudades de España, a principios de siglo, achacándole a veces a tal práctica el interés de los corresponsales por remitir noticias sensacionales y que dieran mucho de sí, como argumentaba El Carbayón a principios de siglo (“Comentarios / Lecciones", 24-10-1901). Pero también pueden documentarse salarios relativamente altos para una actividad que rara vez dejaba de ser el complemento de otras ocupaciones periodísticas. Ya en los años veinte Joaquín Alonso Bonet cobró en algunos momentos la respetable cantidad de 150 pesetas por sus colaboraciones en un conocido rotativo de La Habana, El Diario de la Marina, siendo en 1932 restituido como corresponsal en Gijón del mismo diario con un sueldo mensual de 125 pesetas $^{11}$.

${ }^{10}$ En los años treinta los colaboradores de más prestigio no cobraban más de 50 pesetas por artículo en los periódicos asturianos. Por su parte, todavía en 1936, El Carbayón "pagaba a muchos colaboradores cinco durinos por cada crónica" (Casariego, 1972: 164).

${ }^{11}$ MPA, fondos J. A. Bonet (correspondencia con membrete del Diario de la Marina, 10 y 16 de agosto de 1926 y 5 de abril de 1932). 
Con ello el periodista añadía a sus ingresos habituales como director del diario $\mathrm{La}$ Prensa unas cantidades que económicamente le dejaban en una posición aceptable. Sabemos que en 1923 y en 1924 Bonet había percibido más de 500 pesetas mensuales por dirigir el periódico ${ }^{12}$, suma en torno a la que también se situaba por entonces el sueldo de Antonio López Oliveros al frente de El Noroeste. Unas retribuciones elevadas si se comparan con el pasado sin tener en cuenta otros factores y a condición de no atender más que al ámbito de las redacciones, pero exiguas también en este terreno si se añade por ejemplo que el sueldo de Oliveros, como él mismo señala, se situaba por debajo de lo que "percibía de jornal ordinario y extraordinario el obrero regente de nuestros talleres" (López Oliveros, 1935: 168). Abundan en los testimonios de periodistas de aquellos años las comparaciones desfavorables en el orden económico con los trabajadores de los talleres, cuya temprana organización contrasta, como tantas veces se ha repetido, con la tradicional desunión de los periodistas. Por otra parte, sin ser oficialmente director, aunque sí gobernara el diario desde su posición de Consejero delegado de la empresa editora de El Carbayón, también ascendía a 500 pesetas el sueldo mensual del canónigo Maximiliano Arboleya, con lo que escribía el artículo de fondo y otras dos o tres colaboraciones semanales (Benavides, 1973: 539549). Fue así desde agosto de 1931, asumiendo cuando estaba a punto de nacer el nuevo régimen la dirección del otro gran periódico conservador ovetense, Región, Antonio Álvarez Solís, que poco tiempo después recibía por ello 600 pesetas mensuales ${ }^{13}$.

\section{3. ¿Una verdadera profesión?}

Con unos sueldos escasos, sobre todo en los estratos inferiores del oficio, el pluriempleo se imponía como una necesidad inexcusable. La combinación de actividades en periódicos cuyos intereses no chocaran directamente entre sí -el redactor de un diario asturiano que es al mismo tiempo corresponsal de una o más cabeceras asentadas fuera de la provincia, por citar sólo un caso muy repetido- lejos de constituir un remedio eficaz se convertían en un débil paliativo de las estrecheces sufridas. Mejores expectativas podían concebirse poniendo la vista fuera de las redacciones, en sus aledaños o en profesiones compatibles con el oficio de periodista. Un ejemplo digno de interés e ilustrativo del primer caso en Asturias es el de José Valdés Prida: director de periódicos gijoneses (especialmente de $\mathrm{El} \mathrm{Musel),} \mathrm{corresponsal} \mathrm{literario} \mathrm{y} \mathrm{administrativo} \mathrm{de}$ un gran diario madrileño, coordinador del reparto de numerosas publicaciones periódicas en Gijón, promotor de un proyecto para la instalación de distintos quioscos destinados a la venta de prensa en la ciudad..., todo ello, junto a otras actividades, en los años finales del siglo XIX y los principios del XX, ingresando hacia 1908 en El Noroeste, donde sería redactor, redactor jefe y director (Rodríguez Infiesta, 2007: 407 y ss. 2008: 280-281). De lo segundo, de periodistas que además de serlo desempeñan otras ocupaciones profesionales, están los archivos y las hemerotecas llenos. Ello comportaba evidentes riesgos para la independencia del informador. Poco a poco puede

12 “Honorarios percibidos por los empleados...". Las cantidades anuales ascendieron a 6.050 pesetas en 1923 y 6.500 en 1924.

${ }^{13}$ Casariego, 1972: 164. Cfr., para el momento en que el periodista pasó a dirigir Región, Álvarez Solís, 1946: 157. 
constatarse cómo este tipo de situaciones dejan de ser tan frecuentes, aunque todavía en 1906 Adeflor, que después se convertiría en uno de los más claros ejemplos de periodista completamente profesional, era simultáneamente profesor de una academia de segunda enseñanza (El Noroeste, 18-3 y 5-7-1906). Y todavía en las primeras décadas del siglo los directores de algunos diarios compatibilizaban sus quehaceres periodísticos con la docencia profesional. Tal era el caso de Marcelino Fernández y Fernández, director del modesto diario carlista ovetense Las Libertades, o de Miguel Adellac, durante un breve lapso máximo responsable de un diario de mayor peso, El Noroeste, y que como el anterior era al mismo tiempo profesor de instituto (El Carbayón, 9-111908; Uría: 2004: 202). Habida cuenta de que el cierre de las ediciones se situaba en torno a las cinco de la mañana o un poco antes, parece evidente que una presencia continuada al frente del matutino no tendría fácil concordancia con las otras obligaciones profesionales de aquellos directores. Pronto, en todo caso, se hicieron necesarios periodistas que dirigieran los principales rotativos asturianos a jornada completa, y la vida toda de las redacciones comenzó a regularizarse, requiriendo mayor dedicación por parte de los empleados del periódico.

Ello no siempre implicó que las condiciones de trabajo de los periodistas se plasmaran por escrito. Ni siquiera en los grandes periódicos madrileños como $A B C$, destacado por pagar salarios elevados y promover un periodismo más acorde con los nuevos tiempos, tenían porqué formalizarse las relaciones laborales mediante un contrato de trabajo formal. Tales prácticas, aferradas a un rancio modo de entender la profesión, podían ser vistas todavía como un rasgo de la caballerosidad atribuida al principal propietario de la empresa periodística. Así se refería Alberto Insúa a los resultados de la entrevista que mantuvo con Torcuato Luca de Tena al poco tiempo de iniciarse la Guerra Mundial: "Quedó concertado que yo le enviaría dos o tres crónicas a la semana para $A b c$ y algún que otro artículo, con ilustraciones, para Blanco y Negro. No hubo contrato bilateral. Don Torcuato, al día siguiente de nuestra conversación, me mandó una carta, con las condiciones y precio de mi colaboración. Y esta carta equivalía a una escritura" (Insúa, 2003: 248). Hasta 1926 no se estableció el contrato obligatorio para los integrantes de la plantilla y colaboradores habituales, si bien la normativa no siempre se llevó a la práctica inmediatamente. Se producía tal avance en el marco político de la dictadura de Primo de Rivera, un tiempo durante el que también se establece sobre bases más sólidas que en el pasado el descanso dominical (regulado ya en 1904 y 1920, en esta última fecha con bastante éxito en Asturias ${ }^{14}$ ), se organiza la expedición de carnés de periodista y se ponen en marcha comités paritarios de prensa en distintos puntos de España. Unas mejoras que, sin embargo, fueron

${ }^{14}$ Además de aplicarse la ley de forma mucho más efectiva en 1920, como puede comprobarse en las colecciones hemerográficas asturianas, en Gijón se constituyó un Comité Local de Defensa del Descanso Dominical de la Prensa, presidido por José Valdés Prida, que vinculado al organismo madrileño homólogo trataba de evitar que las disposiciones legales volvieran a quedar en papel mojado. Una copia del compromiso escrito para el respeto a la ley del descanso dominical, firmada por varios periodistas, se conserva entre los papeles de J. A. Bonet del MPA). 
hábilmente utilizadas para el control de la profesión ${ }^{15}$. Para que se diera un contexto más favorable a los profesionales de la información habría que esperar al advenimiento de la Segunda República, creándose por entonces los jurados mixtos, cuyo principal mérito, según se ha señalado repetidamente, consistió en garantizar tanto una remuneración mínima como la protección social y en mejorar la defensa de los asalariados frente a la fuerza de las empresas periodísticas (Desvois, 1996: 42; Ríus Sanchís, 2000: 119 y ss.). Aspectos como las vacaciones pagadas durante un periodo de tiempo de quince, veinte o treinta días, todo un sueño para los periodistas que habían vivido el principio del siglo, comenzaron a ser realidad en los años veinte y treinta. En las "Bases del contrato de trabajo que presentan los periodistas al Jurado Mixto" 16 , se apuntaba un periodo de vacaciones de un mes para los periodistas con al menos un año al servicio del periódico, pudiendo la empresa interrumpir las vacaciones sólo por causas excepcionales, pero debiendo entonces indemnizar a su empleado con un mes de sueldo. En el mismo lugar se conserva copia de un "Proyecto de bases para el contrato de trabajo entre periodistas y Empresas", fechado en Bilbao en 1928, cuyas propuestas no desmerecen de las anteriores. Nada que ver, en todo caso, con la situación que refleja Adeflor a punto de concluir el siglo XIX:

"Los pobres periodistas comenzamos nuestras modestísimas vacaciones, de ocho días a todo más, cuando los veraneantes de alta y baja condición regresan a sus lares. Somos como las golondrinas [...]”. Lo curioso de la cuestión es que las tales vacaciones ni siquiera lo eran del todo, ya que se convirtieron en materia prima de una crónica viajera remitida al periódico a diario (El Noroeste, 22-9-1899 y ss.)

Tenemos, así pues, una profesión que durante mucho tiempo no es tal; que es, principalmente, un oficio mal pagado, lo que conduce al pluriempleo, la bohemia y las más depuradas formas de corrupción. En distintos factores, desde la escasa conciencia de clase que se registra en las redacciones a la elevada exposición a la violencia, se producen avances sensibles durante los años que nos ocupan, lo que por supuesto no elimina inconvenientes de la profesión que podríamos denominar estructurales, comenzando por el cuestionamiento ético del trabajo desarrollado. Ello es fácilmente explicable cuando no concuerda la posición personal del periodista con lo que defiende sobre el papel. No se trata sólo del caso sobradamente conocido y documentado del periodista mercenario, cuyas posiciones políticas se amoldan al diario que le paga. Un cuento del asturiano C. Martínez Riestra, "El tema obligado", publicado originalmente en 1930 y recogido posteriormente por Constantino Suárez (1991: 255-262), puede ilustrar las honduras de ciertos dilemas. Se trata en este caso de un periodista alcohólico obligado a escribir un artículo de fondo contra el abuso de la bebida, representando una "farsa demasiado impropia para sus convicciones de escritor honrado", lo que le lleva al borde de la tragedia. La ausencia de una formación específica previa al ejercicio profesional dificulta, por otra parte, el desarrollo de algún tipo de

${ }^{15}$ Un amplio resumen de las condiciones laborales de la profesión y la acción del Estado durante la época en Ríus Sanchís, 2000: 57 y ss.

${ }^{16} M P A$, fondos J. A. Bonet, hoja fechada a mano en abril de 1932 y redactada unos meses antes. 
compromiso deontológico. Distintas voces abogan por la puesta en funcionamiento de una escuela de periodistas y se dan tímidos intentos para facilitar la enseñanza de algunas habilidades en este campo, pero habría que esperar hasta 1926 para ver en marcha una institución digna de este nombre en España, la denominada Escuela de $E l$ Debate, que aunque ligada a una empresa privada contribuyó a desterrar la sonrisa que durante años había provocado la simple mención de una hipotética carrera universitaria de periodismo. El sentimiento con el que era comúnmente acogida la idea de una facultad de periodismo se plasma incluso en los escritos que propugnaban su implantación. Así se abordaba el tema a principios de siglo en una revista gijonesa: "Existe una Universidad en los Estados Unidos en la que, entre la variedad de carreras que en su seno se estudian, figura, no os riáis, la de periodista. ¿Pero la del periodista es una carrera? Me preguntaréis burlonamente. Y con toda la seriedad que el caso requiere os contestaré. -Sí." (Gerardo Requejo Velarde, "Una idea", Páginas Escolares, 29-6-1904).

\section{Ser periodista... ¿para qué?}

Así planteadas las cosas hay que preguntarse, por lo tanto, qué podía impulsar a alguien a querer formar parte de un gremio como aquel. Es evidente que cuestiones como la escasa exigencia en cuanto a la formación recibida, reflejo de la debilidad del periodismo hispano, podían convertirse en un imán para algunas personas, y otro tanto puede decirse del poder ligado al control de cierta información. No es que ello fuera privativo de la época ni del oficio, pero las prácticas corruptas germinarían con mayor facilidad en el terreno abonado de un oficio pobremente retribuido. Es conocida la gran variedad de prácticas rentables a las que podía recurrir un periodista en la España del siglo XIX y principios del XX, desde el bombo remunerado a las distintas variantes de relación con el poder (con un lugar preferente para el denominado fondo de reptiles). Esto si es que los redactores de un determinado diario no cobraban directamente de las dependencias del Estado, como se aseguró en algún momento que sucedía con el órgano del conservadurismo asturiano La Opinión de Asturias (El Noroeste, 16-3-1900). La cercanía al mundo de la política en sus distintas vertientes era también un factor atrayente. Las posibilidades de promoción social ofrecidas por el periodismo son universalmente conocidas. En España el Congreso siempre estuvo repleto de periodistas, bajo uno u otro régimen, desde principios de siglo (Román Portas, 1999) hasta la república de periodistas a la que alude Checa Godoy (1989: 17-21). En Asturias -ciñéndonos al primer tercio del siglo XX- la prensa sirvió como trampolín para ocupar determinados cargos y a menudo las candidaturas electorales estuvieron más nutridas de periodistas que de ninguna otra profesión. Ramón Álvarez García, Senén Rendueles, Marcelino Trapiello, José Valdés Prida, Marcelino Fernández..., son todos ellos directores o redactores jefe de diarios asturianos que dieron el salto a la política en algún momento, a veces sin abandonar sus responsabilidades periodísticas ${ }^{17}$.

${ }^{17}$ Ramón Álvarez García y Senén Rendueles inician su carrera política al salir elegidos como concejales, en candidaturas distintas, en las elecciones municipales de 1901 en Gijón, siendo el primero director de El Noroeste y el segundo director de El Eco Nacional. Marcelino Trapiello, al poco tiempo de dejar de ser director nominal de El Carbayón, y sin que por ello se 
Había también otros incentivos para entrar en el oficio, comenzando por el disfrute de ciertas prerrogativas o la modesta notoriedad pública aneja al oficio, modestia que no obstante debió ser suficientemente atractiva para muchos jóvenes. El brillo del nombre propio escrito en letras de molde podía constituir un atractivo más poderoso que cualquier otra consideración. "Cuando contemplé mi firma por vez primera en un diario -afirma Antonio Álvarez Solís aludiendo a El Carbayón-, las calles del pueblo eran estrechas para mí. En 25 años de vida profesional, dirigiendo periódicos desde que cumplí 28, no he vuelto a sentir una satisfacción semejante" (Álvarez Solís, 1946: 13). Otro escritor recordaba de forma muy similar su primera colaboración -una necrológica- en el mismo diario ovetense de la mano de Fermín Canella: "Cuando después de leerlo diez o doce veces, salí calle de Campomanes abajo, me parecía que las gentes, asombradas, se fijaban en mí y que hasta deseaban morirse para que yo entonase un himno a su memoria" ("Desde Pravia. Cuartillas de «Pepe García»", El Carbayón, 3-10-1913).

La vía de entrada era siempre la misma, un pariente con amistades en el diario o el propio aspirante a periodista, que sin más títulos que su buena disposición se armaba de valor y proponía o entregaba unas cuartillas manuscritas a quien podía publicarlas. Por supuesto, nada de cobrar en los primeros momentos. Eso vendría más tarde, una vez pasado un aprendizaje que a menudo se hacía bajo la categoría de meritorio, y que le interesaba al periódico más por ahorrarse un sueldo que porque fuera necesario aprender muchas cosas. Aun cuando no faltaran momentos de cierta intensidad cuando se producían determinados acontecimientos, la mayor parte del trabajo de cualquier redactor consistía en recortar fragmentos de otros periódicos y encajarlos en el propio, hinchar telegramas (reconstruir su sentido), recoger lo esencial de las conferencias telefónicas concertadas con una agencia de prensa más tarde... Un tipo de actividad que invitaba a la falta de rigor y hasta a la invención de alguna noticia, hecho rara vez confesado pero sin duda relativamente frecuente y del que puede recogerse algún testimonio. Transcurridos muchos años y tras el velo del seudónimo un periodista escribía desde Oviedo sobre la "innegable utilidad" periodística del infundio y concluía reconociendo "[...] haber ideado una crisis para suplir la sección telegráfica que en determinado día dejó de enviarnos nuestro diligente corresponsal en Madrid, y en ella dar por dimitido al entonces ministro de hacienda, señor Cos Gayón" ( $E l$ Carbayón, 23-9-1922). A fin de cuentas, una proporción muy importante de cualquier periódico la hacían periodistas ajenos a la redacción, o sencillamente personas ajenas al oficio, dentro de la categoría, por ejemplo, de "amigos de la casa" entre quienes, recordaba Constantino Cabal (El Carbayón, 28-8-1915) "había quienes llevaban al periódico las noticias de bodas; y quienes las notas necrológicas; y quienes los relatos de las fiestas de iglesia..."

desvinculara en absoluto del periódico, fue elegido diputado provincial en 1915. José Valdés Prida, al que ya se ha aludido, fue repetidamente concejal del Ayuntamiento de Gijón. Marcelino Fernández fue director del diario carlista Las Libertades y después concejal (reformista) y alcalde de Oviedo. 
Los límites del oficio de periodista no son, en todo caso, fáciles de precisar en esta época, lo que supone una dificultad añadida a cualquier intento de acercamiento cuantitativo a la cuestión. No disponemos de un censo profesional para toda la región, aunque la nómina sería sin duda extensa teniendo en cuenta que cualquier núcleo de población de mediano tamaño contaría al menos con los corresponsales de los principales diarios asturianos. En 1930 eran como mínimo cuatro los corresponsales que desde Candás informaban a distintos periódicos ${ }^{18}$; y algunos años antes, en 1924, tenían corresponsal en otra pequeña población, Grado, los diarios La Voz de Asturias, El Carbayón, Región, El Comercio, La Prensa y El Noroeste (Gordero Chamorro, 1924). Periodistas a tiempo parcial, de modo que ni siquiera uno de ellos, el citado Gordero Chamorro, que era simultáneamente corresponsal en Grado de El Carbayón, El Sol, La Voz y la Agencia Febus -los tres últimos estrechamente ligados- debía obtener por esta actividad más que un pequeño suplemento a su sueldo como maestro nacional; pero periodistas al fin y al cabo.

La definición de periodista puede aparecer también en otras ocasiones, por ejemplo en los censos electorales; que ello se utilice como elemento definidor preferente puede resultar significativo, aunque sigue siendo muy difícil distinguir si tras el término se oculta una actividad mínimamente profesionalizada ${ }^{19}$. Muy distinto es lo que sucede en las grandes poblaciones asturianas donde la aparición de nombres sobradamente conocidos confirma una dedicación profesional más sólida en términos generales. El número de periodistas registrados como tales en Gijón en 1924 asciende a diecisiete según la Guía Oficial y Profesional de la ciudad editada por Antonio Pérez Pimentel (1924: 89). Las estadísticas de la prensa promovidas cada siete años desde 1913, aunque a diferencia del caso anterior omiten nombres y apellidos, no contradicen tales datos al señalar dieciocho redactores de plantilla en los diarios gijoneses en 1927. En la totalidad de los diarios asturianos se contabilizan 34 redactores de plantilla en 1913, 29 en 1920 y 45 en 1927; y si a esta categoría incorporamos los "colaboradores a sueldo" (sin una casilla específica en 1913), tendríamos 40 en 1920 y 63 en $1927^{20}$. El problema principal está, una vez más, en saber en qué medida estamos refiriéndonos a profesionales asalariados. A otro tipo de hipotéticas distorsiones se añade en este caso la duda sobre los redactores de plantilla, de los que parece razonable considerar que sean asalariados, al menos en los diarios, aunque debe tenerse en cuenta que algunos pequeños semanarios indican cifras muy elevadas en este apartado. En todo caso, parece que en las primeras décadas del siglo XX ya es posible referirse a un núcleo reducido y relativamente estable de profesionales del periodismo en Asturias.

Por otra parte, no debe olvidarse que puede entender el asociacionismo, entre otras cosas, como síntoma de ciertas inquietudes y de la existencia de un creciente grupo de

${ }^{18}$ Archivo Histórico de Carreño, 335/2 ("Relación nominal detallada y clasificada de entidades profesionales y particulares que se presume interesadas a contribuir a la organización de los festejos con las cantidades que se expresan y demás obsequios", 1930).

${ }^{19}$ Véase como ejemplo, para Noreña en 1910, la mención recogida en Rodríguez Buznego, 2006: 24.

${ }^{20}$ Estadística de la prensa periódica de España, 1914: 80-81; 1921: 68-71; 1930: 72-75. 
personas ocupadas en las actividades periodísticas. Fue durante el verano de 1906 cuando comenzó a aludirse a la posible creación de una "Asociación regional de periodistas y escritores". Tras surgir la idea en una reunión de periodistas ovetenses en la que estaba presente un colega madrileño (la Asociación de la Prensa de Madrid se había fundado en 1895), distintos diarios acogieron con simpatía la iniciativa, se impulsó la creación de una comisión provisional organizadora y se esbozaron los posteriores pasos a seguir ${ }^{21}$. Unos meses más tarde se proponía la creación de una Asociación de la prensa (El Carbayón, 21-12-1906). Sin embargo nada de aquello tomó forma definitiva, y al año siguiente volvía a saltar la chispa de la mano de Protasio González Solís, uno de los más eminentes nombres de la historia del periodismo astur, que se había dirigido ya meses atrás a la prensa abogando por la asociación y volvía a insistir en agosto de 1907 (El Carbayón, 6-8-1907). Cuajó algunas semanas más tarde la idea de un banquete que serviría como acto constitutivo de la Asociación de la prensa asturiana; pero una vez más todo aquello no respondió a las expectativas levantadas, ya que finalmente fue a finales de 1909 cuando se creó la primera de las asociaciones de la prensa asturianas: la de Gijón, con carácter local. Tras fracasar la propuesta de que esta última se convirtiera en organización regional, no tardó demasiado tiempo en constituirse en Oviedo una asociación análoga, desarrollando ambas distintas actividades en los años siguientes ${ }^{22}$.

Sin embargo, la convivencia entre socios con intereses muy distintos hizo que pronto afloraran las diferencias, de manera que de la asociación gijonesa surgió la idea de crear una "Sociedad de periodistas profesionales" ya en 1914. "Es tan distinta la cualidad de periodista profesional de la que representan aquellos otros señores asociados para quienes esta condición es sólo un accidente ocasional o pretérito -se afirmaba al respecto-, que tal demostración no requiere mayores explicaciones" ( $E l$ Noroeste, 6-8-1914). Las dos principales asociaciones asturianas tuvieron muy presente en sus reglamentos lo que significaba formar parte de la plantilla de un periódico, recibir un salario o, como señalaban los estatutos de la Asociación de la Prensa Diaria de Gijón en 1925 "tener como base principal de vida el periodismo". Por otra parte, en los años veinte y treinta llegaron a existir en Asturias distintas sociedades de periodistas que por una u otra razón utilizaron el calificativo "profesional" en su denominación ${ }^{23}$. Se había pasado, en definitiva, de considerar factible la convivencia de

${ }^{21}$ Véase, por ejemplo: El Carbayón, 18-8-1906 y 25-8-1906; El Noroeste, 22-8-1906; El Popular, 23-8-1906.

${ }^{22}$ La historia de la Asociación de la prensa ovetense en estos años y el nacimiento de la de Gijón, así como los precedentes inmediatos (es decir, el citado banquete y sus derivaciones), pueden seguirse en el detallado trabajo de Fernández Martínez, 2011. La propuesta aludida, especialmente en: Roque, “Asociación de la Prensa", El Carbayón, 29-11-1909.

${ }^{23}$ En el Archivo Histórico de Asturias, Fondo Asociaciones, Gobierno Civil, exp. 20082/08 pueden consultarse los reglamentos de la Asociación de la Prensa Diaria Ovetense y el reglamento de la Agrupación Profesional de Periodistas de Gijón de 1927 (exp. 20063). Véanse también los apéndices a: Pereira Mon, 2004 (pp. 583-604), donde además se recogen los estatutos de la Asociación de la Prensa Diaria de Gijón. Para otras organizaciones: Fernández Martínez, 2011: 45. 
escritores y periodistas en un mismo colectivo a distinguir claramente a quienes desarrollaban una actividad profesional en el campo del periodismo.

\section{Conclusiones}

Todo ello apunta, una vez más, al lugar central que ocupa la época analizada cuando se trata de comprender el despegue hacia una situación en la que ya es posible aludir claramente a profesionales del periodismo, lo que se plasma en la necesidad de defender unos intereses comunes, en el aumento progresivo de los salarios o en las plantillas que los diarios tienen a su disposición. Ello no significa que no se mantengan, incluso en los años treinta, algunos de los rasgos de un periodismo amateur y poco menos que familiar, como se había practicado en Asturias durante el siglo anterior. El periodista sigue siendo un trabajador peculiar, que se codea con las clases altas y con los protagonistas de la actualidad, pero que a menudo contempla con envidia las condiciones laborales y los salarios de los obreros, acentuándose lo delicado de su situación si tenemos en cuenta que, como indicaba Adeflor (El Noroeste, 6-9-1907) "al periodista se le exige vivir, por razones de su profesión, en un medio social superior a sus fuerzas económicas".

Como es lógico, las diferentes categorías que se establecen desde el corresponsal en una pequeña población hasta el redactor jefe o director condicionan las diferencias de un oficio en el que la formación específica es casi nula y que, en términos generales, se considera mal pagado, lo que sin duda está en el origen de muchas de las actividades poco confesables que se les reprochan a los periodistas. El pluriempleo, que en los primeros años analizados se da incluso en la dirección de algunas cabeceras, va dejando paso a una actividad que cada vez se va haciendo más difícilmente compatible con otras ocupaciones cuando nos referimos a las más altas responsabilidades de los principales diarios. De todos modos, la prensa asturiana no puede ser estudiada como un caso aislado, sino en relación con otras realidades regionales y provinciales y teniendo presente la influencia de la prensa madrileña de difusión nacional, siempre presente.

\section{Referencias bibliográficas}

ÁLVAREZ SOLÍS, Antonio (1946): Desde el Polo Norte. Anecdotario, recuerdos y divagaciones de un periodista. Barcelona, Editorial Vives.

BENAVIDES GÓMEZ, Domingo (1973): El fracaso social del catolicismo español. Arboleya-Martínez 1870-1951. Barcelona, Nova Terra.

BLANCO, Santiago (1977): El inmenso placer de matar un gendarme. Memorias de guerra y exilio. Madrid, Cuadernos para el Diálogo.

CABAL, Constantino (1941): Nombres de Asturias. Don Fermín Canella. Oviedo, Escuela Tp. de la Residencia Provincial.

CAMBA, Julio (1946) [1ª edic. 1927]: "Sobre las huelgas periodísticas", en CAMBA, Julio: Sobre casi todo. Madrid, Espasa Calpe, pp. 24-27.

CASARIEGO, Jesús Evaristo (1972): "Periódicos asturianos: su número, clase y distribución”. Boletín Del Real Instituto De Estudios Asturianos, no 75, pp. 159-174. 
CASARIEGO, Jesús Evaristo (1990): "D. Joaquín A. Bonet y el periodismo". VV.AA.: Don Joaquín Alonso Bonet. Vida y obra de un hombre de letras asturiano 1889-1975. Oviedo, IDEA, 1990, pp. 17-52.

CHECA GODY, Antonio (1989): Prensa y partidos politicos durante la II República. Salamanca, Ediciones Universidad de Salamanca.

DESVOIS, Jean-Michel (1996): "El estatus de periodista en España de 1898 a 1936: Nacimiento y consolidación de una profesión". Valencia, Comunicación y Estudios Universitarios, $\mathrm{n}^{\circ}$ 6, pp. 33-46.

Estadística de la prensa periódica de España (Referida al $1^{\circ}$ de abril del año 1913) (1914). Madrid: Ministerio de Instrucción Pública y Bellas Artes. Dirección General del Instituto Geográfico y Estadístico.

Estadística de la prensa periódica de España (Referida al $1^{\circ}$ de febrero del año 1920) (1921). Madrid: Ministerio de Instrucción Pública y Bellas Artes. Dirección General del Instituto Geográfico y Estadístico.

Estadística de la prensa periódica de España (Referida al 31 de diciembre de 1927) (1930). Madrid: Ministerio de Trabajo y Previsión. Servicio General de Estadística.

FERNÁNDEZ MARTÍNEZ, Georgina (2011): "La Asociación de la Prensa Diaria Ovetense en el primer tercio del siglo XX". VV.AA., 1911- 2011. Asociación de la Prensa de Oviedo. Un siglo de periodismo. Oviedo, Asociación de la Prensa de Oviedo.

GARCÍA VENERO, Maximiano (1961): D. Torcuato Luca de Tena y Álvarez-Ossorio. Una vida al servicio de España. Madrid, Editorial Prensa Española.

GÓMEZ MOMPART, Josep Lluís (1992): La gènesi de la premsa de masses a Catalunya (1902-1923). Barcelona, Pórtic.

GORDERO CHAMORRO, José (1924): Álbum comercial, literario, gráfico, descriptivo de Grado y su concejo. Oviedo (reed., Oviedo, 2007).

Guía Oficial y Profesional de Gijón 1924 (1924): Gijón, Imprenta La Victoria.

INSUA, Alberto (2003): Memorias. Madrid, Fundación Santander Central Hispano, 2003.

LAGUILLO BONILLA, José (1979): Memorias. Veintisiete años en la dirección de El Liberal de Sevilla (1909-1936). Sevilla, Secretariado de Publicaciones de la Universidad de Sevilla.

LÓPEZ OLIVEROS, Antonio (1935): Asturias en el resurgimiento español (Apuntes históricos y biográficos). Madrid (reed. Gijón, 1989).

PEREIRA MON, Ana Celia (2004): "El asociacionismo periodístico en Asturias y los primeros pasos de la Asociación de la prensa de Oviedo (1909)", en URÍA, Jorge (coord.): Historia de la prensa en Asturias. I Nace el cuarto poder. La prensa en Asturias hasta la Primera Guerra Mundial. Oviedo, Asociación de la Prensa de Oviedo, pp. 521-533. 
RADCLIFF, Pamela Beth (2004): De la movilización a la Guerra Cicil. Historia política y social de Gijón (1900-1937). Barcelona, Debate.

RÍUS SANCHÍS, Inmaculada (2000): El periodista, entre la organización y la represión: 1899-1940. Para una historia de la Asociación de la Prensa Valenciana. Valencia, Fundación Universitaria San Pablo C.E.U.

RODRÍGUEZ BUZNEGO, Oscar (2006): Elecciones en Noreña. Noreña, Contigo.

RODRÍGUEZ INFIESTA, Víctor (2007): Socialización politica y prensa de masas. El proceso de la opinión pública en Asturias, 1898-1923. Oviedo, Real Instituto de Estudios Asturianos.

RODRÍGUEZ INFIESTA, Víctor (2008): "La distribución de la prensa diaria en los inicios del siglo XX: el espacio asturiano". Zer. Revista de Estudios de Comunicación, Bilbao, vol. 13, n 25, pp. 269-286.

ROMÁN PORTAS, Mercedes (1999): "Periodistas y políticos. El Congreso de los Diputados en 1901 y en 1996", en: BARRERA, Carlos (coord.): Del gacetero al profesional del periodismo. Evolución histórica de los actores humanos del "cuarto poder". Madrid, Fragua.

SANTULLANO, Gabriel (2004): "El oficio de periodista en el siglo XIX”, en URÍA, Jorge (coord.): Historia de la prensa en Asturias.... . Oviedo, Asociación de la Prensa de Oviedo, pp. 455-491.

SEOANE, $\mathrm{M}^{\mathrm{a}}$ Cruz y SÁIZ, M ${ }^{\mathrm{a}}$ Dolores (1996): Historia del periodismo en España 3. El siglo XX: 1898-1936. Madrid, Alianza.

SUÁREZ, Constantino (1991): Cuentistas Asturianos. Gijón, Editorial Auseva.

URÍA, Jorge (2004): "El radicalismo democrático de El Noroeste", en URÍA, Jorge (coord.) Historia de la prensa en Asturias... . Oviedo, Asociación de la Prensa de Oviedo pp. 199-239.

VALLADARES FERNÁNDEZ, Edelmiro (1996): Historia de una empresa periodística gallega: LA REGIÓN. Orense, La Región S.A. 